\title{
Integrated microgiant electrorheological fluid valves for microflow cytometry
}

\author{
Yun-Yang Ling \\ Yechi Zhang \\ Hong Kong University of Science and Technology \\ Department of Mechanical Engineering \\ Clear Water Bay \\ Hong Kong
}

\author{
Weijia Wen \\ Hong Kong University of Science and Technology \\ Department of Physics \\ Clear Water Bay \\ Hong Kong
}

\author{
Patrick Tabeling \\ École Supérieure de Physique et de Chimie \\ Industrielle \\ Microfluidic MEMS and Nanostructures Laboratory \\ 10 rue Vauqueline \\ Paris, 75231 \\ France
}

\author{
Yi-Kuen Lee \\ Hong Kong University of Science and Technology \\ Department of Mechanical Engineering \\ Clear Water Bay \\ Hong Kong \\ E-mail: meyklee@ust.hk
}

\begin{abstract}
We present the fabrication and optimization of new integrated microgiant electrorheological (GER) valves for microflow cytometry. Compared to previous GER valves, new GER valves, consisting of an SU-8 layer, a PDMS membrane, and glass layers, were fabricated by 4-mask microelectromechanical systems technology and two new packaging methods. This design enabled good bonding and fluidic interconnection. The thickness of the PDMS membrane was designed such that the membrane deformation was large enough that the cytometry channel was well sealed. The interfacial stress between the PDMS and the PDMS/SU-8 as a function of vacuum plasma treatment time was investigated in detail. The switching behavior of the GER valves was also analyzed and characterized using fluorescence microscopy. (c) 2009 Society of Photo-Optical Instrumentation Engineers. [DOI: 10.1117/1.3124191]
\end{abstract}

Subject terms: electrorheological fluid; GER fluid; microvalves; SU-8; PDMS.

Paper 08144SSR received Aug. 20, 2008; revised manuscript received Feb. 5, 2009; accepted for publication Mar. 17, 2009; published online May 5, 2009.

\section{Introduction}

The manipulation of biofluids and bioparticles is very important in the microfluidic applications, but it has been extremely difficult to accomplish. Many different microvalves have been designed and reported in attempts to effectively achieve such manipulation. ${ }^{1}$ Usually, these microvalves have been controlled or actuated by piezoelectric elements, ${ }^{2}$ pneumatic solenoid valves, ${ }^{3}$ or hydrogels. ${ }^{4}$ The rapidly developing microelectromechanical systems (MEMS) techniques have made it possible to fabricate and integrate these key components on Lab-on-a-Chip devices, some of which have allowed researchers to build a complicated microvalve system easily on several types of substrates, such as glass, ${ }^{5}$ silicon, ${ }^{6}$ and polymers, ${ }^{7}$ so that some advantages, including lower cost, compact size, and disposability to avoid contamination, could be achieved.

In past several years, Quake's group ${ }^{3}$ has introduced polydimethylsioxane-based (PDMS-based) soft lithography to fabricate micropneumatic valves for large integration of microfluidics by Quake's group. A fluidic multiplexor (i.e., a combinatorial array of binary micropneumatic valves) was proposed to increase fluidic processing power. This novel microfluidic chip consists of the upper channel ("con-

$1932-5150 / 2009 / \$ 25.00$ @ 2009 SPIE trol channel"), the lower channel ("flow channel"), and the membrane of polymer between the channels. Two layers formed by the PDMS rapid prototyping technique were bonded together in a crossed-channel architecture. The bonded structure was sealed on the top of an elastomer substrate. When pressurized gas was applied to the upper pneumatic control channels, the flexible membrane was deformed to block the flow channel at the intersection of the in-line microchannels at the lower layer. This type of microvalve, which is controlled by external, mini-pneumatic, solenoid valves, has received intensive study due to its flexibility, biocompatibility, and ease of fabrication. ${ }^{3}$ Although these valves have some notable advantages, the lowfrequency response of PDMS-based microvalves prevents them from being used in high-speed switching devices, such as microflow cytomters. Most microflow cytometers that use micropneumatic valves have low switching speeds when compared to a traditional flow cytometer. ${ }^{8}$ Therefore, an integrable and digitally addressable valve with fast response time (milliseconds) is still needed in microflow cytometer systems.

Electrorheological fluid (ER fluid) is a type of colloidal suspension that has rheological characteristics and can be controlled through the application of an external electric field. Micro-ER valves made of silicon and glass substrates were first reported by Yoshida et al. ${ }^{9}$ and Volder et al. ${ }^{10}$ The 
ER effect of several liquid crystals in SU-8 microchannels with indium tin oxide electrodes has been tested. However, these devices are designed mostly to control the flow of the ER fluid itself (two-way valve), and they cannot be considered as an integrated microvalve for biofluid manipulation. Wen et al. ${ }^{11}$ successfully developed a new ER fluid, referred to as giant electrorheological fluid (GER fluid). The GER fluid is made of 20-nm urea-coated nanoparticles suspended in silicone oil, which has high shear stress and fast response time (within $10 \mathrm{~ms}$ ), making it an ideal smart material for microfluidic applications. On the basis of this type of ER fluid, we developed a new GER fluid-based microvalve (micro-GER valve, abbreviated as MGV). Practically, these MGVs were developed on both PDMS and conductive PDMS. Conductive PDMS (CPDMS) as the microelectrode material, is a polymer composite synthesized by mixing PDMS and carbon black at a carbon concentration of $25 \%(\mathrm{w} / \mathrm{w})$ to form the so-called CPDMS. Several microGER valves have been fabricated and characterized. The on-off switching time of micro-GER valves was found to be $\sim 8 \mathrm{~ms}^{12}$ Furthermore, these integrated MGVs have also been applied to highly efficient microfluidic mixing and micropumping. ${ }^{13,14}$ Although MGVs have shown their great potential for use in microfluidic applications, they have some limitations [e.g., the fabrication process is quite complicated and time-consuming and manual alignment of the multilayer (PDMS/CPDMS) microfluidic devices under an optical microscope is necessary]. Obviously, these issues would inevitably create uncertainties in microfabrication process. ${ }^{12}$

In this paper, to overcome the difficulties and uncertainties existing in the original fabrication process, we designed and fabricated a new MGV using the four-mask MEMS process, as shown in Fig. 1. This new MGV consists of SU-8 GER control channels on the glass substrate integrated with 3D micrometal electrodes and a sealed PDMS flow cytometer channel. In addition, a new packaging method was developed for this $\mathrm{MGV}$, which resulted in much higher reliability of the device.

\section{Fabrication of Micro GER Valves}

\subsection{Fabrication of 3-D Microelectrodes}

The key fabrication processes of the MGV are summarized in Fig. 2. First, a liftoff process was used to pattern planar microelectrodes. Next, a layer of $0.3-\mu \mathrm{m}$-thick LOL2000 (Shipley Co., MA, USA) was spin-coated at $1000 \mathrm{rpm}$ for $30 \mathrm{~s}$. Then, a layer of $1.2-\mu \mathrm{m}$-thick FH6800L photoresist (Fujifilm Arch Co., Japan) was spin-coated at $4000 \mathrm{rpm}$ for $60 \mathrm{~s}$. After patterning the FH6800L, additional $\mathrm{Ti} / \mathrm{Cu}$ (100 nm/400 nm-thick) layers were sputtered. Therefore, the 500- $\mu \mathrm{m}$-wide planar copper electrodes were formed in an ultrasonic bath of acetone [Fig. 2(b)].

The 3-D microelectrodes were fabricated by a selective electroplating process [Fig. 2(c)]. First, a layer of $40-\mu \mathrm{m}$-thick AZ4620 photoresist was patterned on the wafer to restrict the copper electroplating area (400 $\times 200 \mu \mathrm{m})$. A layer of $30-\mu \mathrm{m}$-thick copper was formed on the restricted area in an electroplating solution (Intervia ${ }^{\mathrm{TM}}$ $\mathrm{Cu}$ 8540, Rohm and Haas, Germany) for $4 \mathrm{~h}$ using a current density of $0.1 \mathrm{~mA} / \mathrm{cm}^{2}$ [Fig. 2(c)]. Finally, AZ 4620

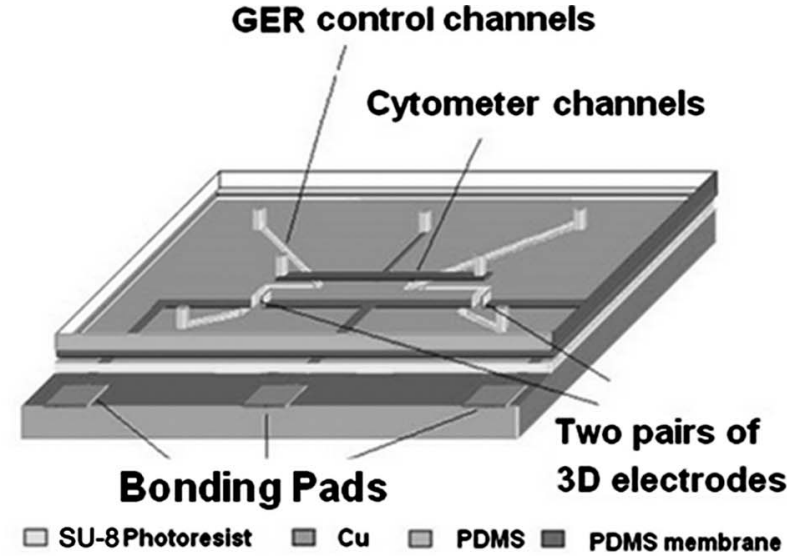

(a)

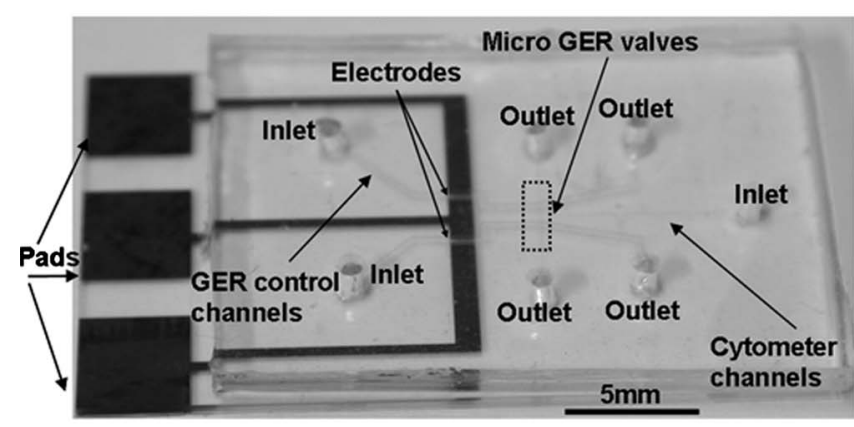

(b)

Fig. 1 (a) Schematic of a microflow cytometer with micro-GER valves. (b) Photograph of the device.

photoresist was stripped off and the wafer with 3-D copper electrodes was baked on a hot plate at $120^{\circ} \mathrm{C}$ for $30 \mathrm{~min}$ for the SU-8 process.

\subsection{SU-8 and PDMS Processes}

A layer of SU-8 2100 photoresist (MicroChem Corp., Masschusetts) was spin-coated on the wafer at $2000 \mathrm{rpm}$ for $30 \mathrm{~s}$ for the GER control channels. After the contact photolithography, the 120 - $\mu \mathrm{m}$-high, 400- $\mu \mathrm{m}$-wide SU-8 microchannels were formed. Similar to our previous work, ${ }^{4}$ AZ 4620 photoresist was employed to form the mold for flow cytometer channels. This photoresist mold was baked at $80{ }^{\circ} \mathrm{C}$ for $30 \mathrm{~min}$ to have a rounded cross section, which can avoid the dead volume of the valve. The height and

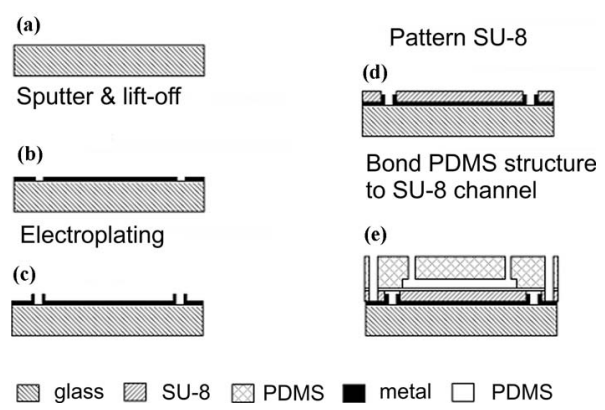

Fig. 2 Simplified fabrication processes for micro-GER valves. 

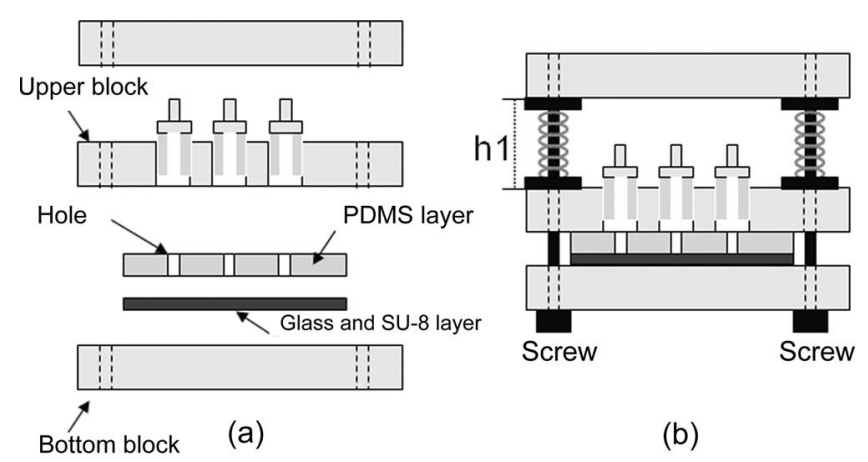

Bottom block (a)

(b)

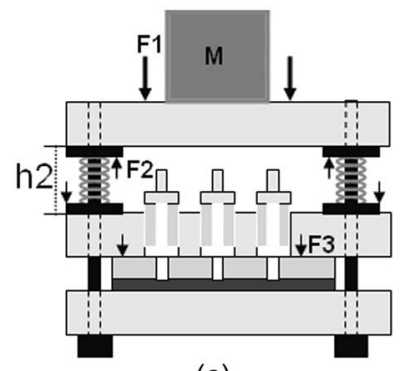

(c)

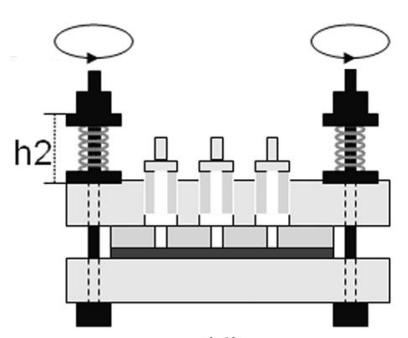

(d)

Fig. 3 Schematic diagrams of the mechanical bonding and packaging processes for a microflow cytometer.

width of the AZ 4620 mold were measured to be $10 \mu \mathrm{m}$ at the top of the cross section and $50 \mu \mathrm{m} / 150 \mu \mathrm{m}$ at T-junction/the rest of flow cytometer channels, respectively. The mixture of PDMS (Sylgard 184, Dow Corning, USA) resin and curing agent at the ratio of 10:1 was casted onto the AZ 4620 mold on a glass wafer.

Another $25-\mu \mathrm{m}$-thick layer of PDMS was spin-coated on a silicon wafer at $3000 \mathrm{rpm}$ for the valve membrane. Both the PDMS membrane and flow cytometer channel layer were cured at $75^{\circ} \mathrm{C}$ for $30 \mathrm{~min}$. The layer of cytometer-channel pattern was peeled off from the mold, and three holes were punched at the both ends of the channels for the inlets and outlets. Finally, the layer of cytometer channel and that of PDMS valve membrane were bonded together to form a temporary PDMS structure. After 2 min of vacuum plasma treatment (PDC-002, Harrick Sci. Corp), the PDMS structure was peeled off from a silicon wafer.

\subsection{SU-8/PDMS Bonding and Packaging}

To completely seal the GER control channel without any leakage under normal valve actuating pressure, two strategies were proposed in our work (i.e., permanent bonding and mechanical sealing of PDMS/SU-8). For the permanent bonding, we activated the surfaces of PDMS and SU-8 by the vacuum plasma treatment, and these two active surfaces induced a surface reaction to form chemical bonding, which was permanent and irreversible. To optimize the bonding of PDMS/SU-8 with the vacuum plasma treatment, a copper handle was glued to some bonded samples using an epoxy adhesive (Araldyte $\AA$, Vantico, Ltd.) and pulled perpendicularly by the Micro-Force Testing Machine (Alliance RT/5, MTS Systems Co., USA) to achieve the maximal interfacial stress between PDMS/PDMS and PDMS/

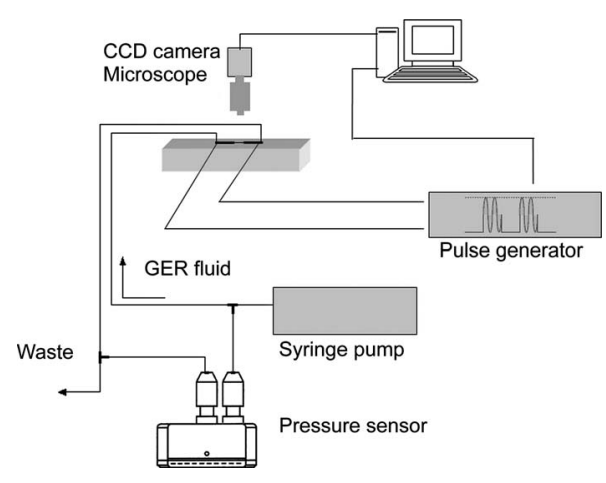

Fig. 4 Experimental setup for micro-GER valves.

SU-8. Experimentally, a vacuum time of $2 \mathrm{~min}$ and a plasma treatment time of $1 \mathrm{~min}$ were used to activate the SU-8 surface.

For the mechanical bonding/packaging process as shown in Fig. 3, first, we attached the PDMS layer on the SU-8 layer. Then, the MGV chip was sealed via a pair of mechanical clamps. The clamps consisted of two 5-mm-thick Plexiglas blocks and four screws located on the four edges of the blocks. The space between the clamps was easily controlled by fastening and releasing the screws with the help of a vernier caliper. Inlet and outlet holes were drilled in the upper part of the block for the cell suspension and the GER fluid. Flexible tygon tubing (with an outer diameter of $3 \mathrm{~mm}$ ) was inserted into the holes and fixed in place with an epoxy glue. Barbed adapters were used for fluidic interconnects.

Because the PDMS material can be easily deformed, the clamping pressure was controlled carefully and accurately. In our packaging process, mechanical springs were used here to control the pressure on the clamps [Fig. 3(b)]. First, we gradually increased the weight on the springs [Fig. 3(c)] and then measured the deformation of the springs. There was a linear relationship between the weight and the deformation of springs, and the total elastic constant $k$ of springs could be readily determined. Because the contact area of PDMS/SU-8 was constant for the MGV chip packaging, we adjusted the screws to the regular position to apply the right correct pressure on the MGV chip. The magnitude of pressure was actually linearly dependent on the change in the length of springs [Fig. 3(d)]. The hermeticity of the interfaces between the PDMS/Plexiglas and the PDMS/SU-8 was examined using pressurized $\mathrm{N}_{2}$ and water. A slight deformation, which was not enough to damage the PDMS channels, ensured that there was no leakage of GER fluid into the GER control channels under the operating pressure.

\section{Membrane Deformation Model}

In order to optimize the design of the device, we used an analytical method to predict the maximum deformation of the PDMS membrane $w_{m}$,

$w_{m}=\frac{0.0284 q a^{4}}{E h^{3}\left[1.056(a / b)^{5}+1\right]}$,

where $h$ is the thickness of the PDMS membrane, $E$ is Young's modulus, $q$ is the pressure applied to the PDMS 
Table 1 Comparison of the theoretical and experimental values of PDMS membrane deformation and the conditions to turn off the valve.

\begin{tabular}{ccc}
\hline \hline & Theory $^{\mathrm{a}}$ & Experiment \\
\hline Membrane deformation & $8.36 \mu \mathrm{m} / \mathrm{psi}$ & $8 \mu \mathrm{m} / \mathrm{psi}$ \\
Critical parameters & $\mathrm{N} / \mathrm{A}$ & $>9 \mathrm{psi}$ pressure or \\
& & $>2000 \mathrm{~V} / \mathrm{mm}$ of e-field \\
\hline \hline
\end{tabular}

The thickness of PDMS membrane is $25 \mu \mathrm{m}$, a and b are 150 and $400 \mu \mathrm{m}$, respectively, and the height of cytometer channels is $12 \mu \mathrm{m}$.

membrane, and $a$ and $b$ are the sizes of the cytometer channel and the GER control channels, respectively. The calculated and experimental values listed in Table 1 show good agreement.

\section{Experimental Setup}

As shown in Fig. 4, the experimental setup for the micro GER valves consisted of two major parts [i.e., (i) the microflow cytometer and a clamp and (ii) the detection and image acquisition system]. The pressure drops along the microchannels were systematically measured by a pressure sensor (Model ASCX 15AN, Honeywell, USA) with a pressure range of 15 psi. A digital syringe pump was used to control the flow rate of the GER fluid. In addition, a pneumatic testing system was also set up to characterize the micro-GER valve under controlled pressure of $\mathrm{N}_{2}$ gas. The $\mathrm{N}_{2}$ pressure was measured by another pressure sensor (Model No. ASCX15DN, Honeywell, CA), which had a maximum detection limit of $15 \mathrm{psi}$. An inverted fluorescent microscope (Olympus IX70, Japan) and a thermoelectrically cooled CCD camera (Retiga 1300C, QImagining Co., Canada) were used for the optical detection of the cells. The CCD camera was also connected to a computer via an IEEE 1394 interface. A LabVIEW image acquisition program was designed to control the parameters of the CCD camera, such as exposure time and digital image storage.

\section{Results and Discussions}

Vacuum plasma treatment is a process that typically operates in the range of $39-120^{\circ} \mathrm{C}$ to avoid thermal damage,

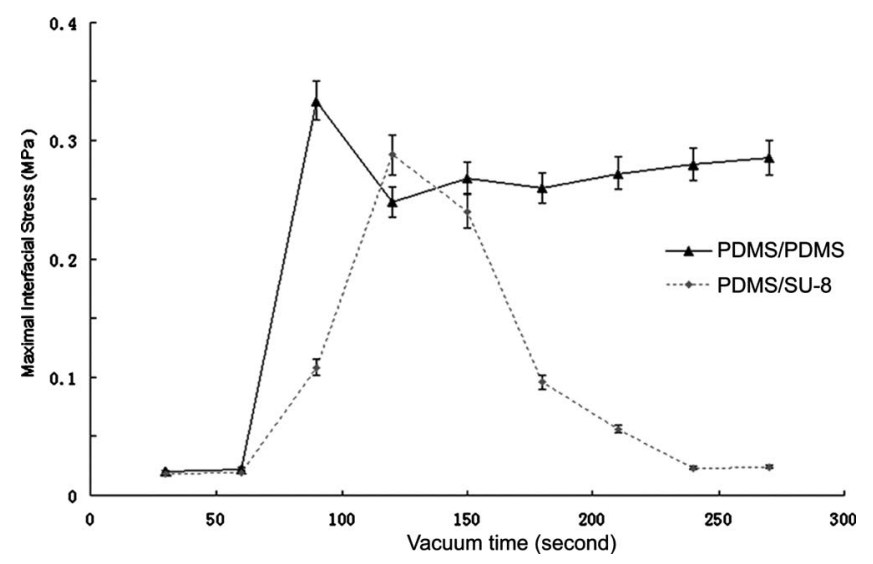

Fig. 5 Maximal interfacial stress as a function of the processing time in a vacuum plasma chamber. and it was used in this work for preparation of the surfaces of SU-8 and PDMS. This process can induce nonthermally activated surface reactions, causing surface changes that cannot occur with molecular chemistry at atmospheric pressure. The plasma also generates electromagnetic radiation in the form of UV photons to penetrate bulk polymers to a depth of $\sim 10 \mu \mathrm{m}$, which can cause chain scissions and cross-linking. ${ }^{16}$ For the activation of the surface of the SU-8 layer, the important parameter (vacuum time using the accessorial vacuum pump) was carefully studied. In the experiments, a set of bonded samples was made by bonding PDMS cubes with a bare SU-8 coated plate (SU-8 photoresist on a glass plate) for which its bonded area was set to $5 \times 5 \mathrm{~mm}$. Their maximal interfacial stress imposed on the samples was recorded to explore the optimized vacuum time for the good bonding of PDMS/SU-8 (Fig. 5). Meanwhile, for the comparison to PDMS/PDMS, the measurement of maximal interfacial stress of PDMS/PDMS was also studied. To obtain a good PDMS/SU-8 bonding, the ranges of 2-2.5 min of vacuum time and $1-2 \mathrm{~min}$ of plasma treatment were found to be effective. Other parameters were found to lead to weak bonding or even bonding failure.

A maximum pressure of 15 psi could be applied to the channels with this bonding method. No leakage between the interface of SU-8/PDMS was observed under the microscope.

Similar testing was carried out on the MGV chip fabricated by the mechanical sealing of SU-8 and PDMS, but the chips was not treated with the vacuum plasma. Thus, there was no permanent bonding between these two layers. Because of the electron affinity (physical) of SU-8 and

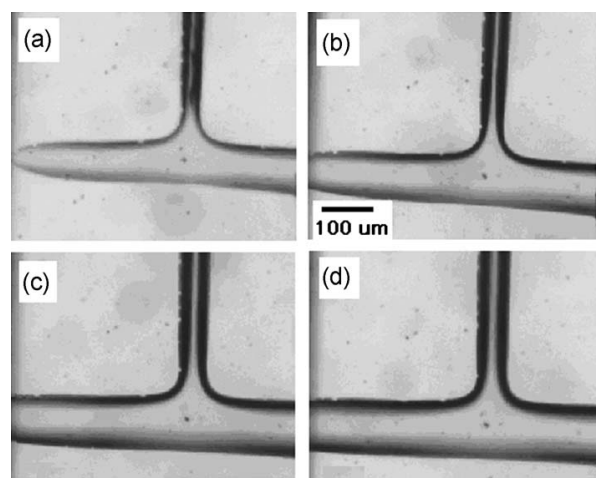

Fig. 6 Optical micrographs of cytometer channel under different applied pressure (A: $5 \mathrm{kPa}, \mathrm{B}: 14 \mathrm{kPa}, \mathrm{C}: 27 \mathrm{kPa}$, and D: $45 \mathrm{kPa}$ ). 


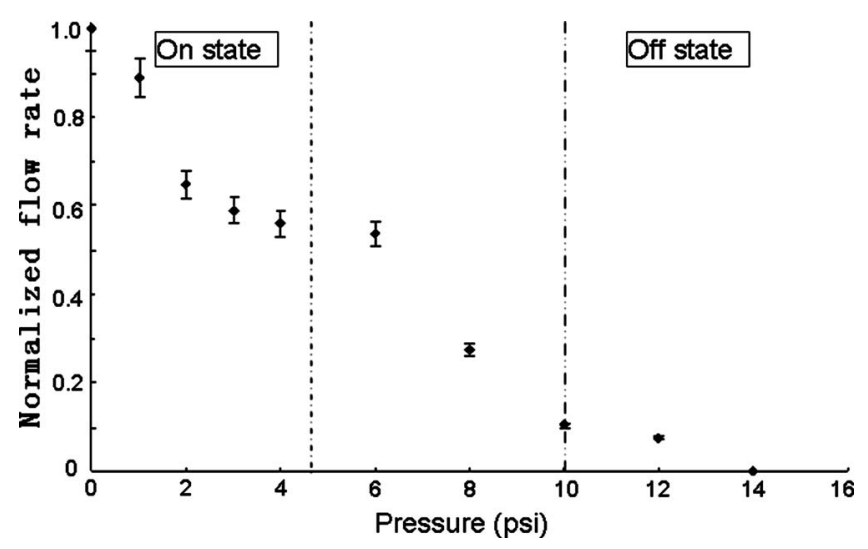

Fig. 7 Flow rate inside the cytometer channel as a function of input pressure inside the GER control channel.

PDMS, the PDMS was easy to attach to the SU-8 layer. After carefully removing the air bubbles, this microchip was sealed with a pair of mechanical clamps that provided a uniform pressure, creating a good mechanical sealing between the SU-8 and the PDMS.

The magnitude of pressure was crucial for the MGV chip packaging. Lower pressures would lead to leakage at the interface, while higher pressures led to serious deformation of the cytometer channel. Therefore, the proper pressure should produce slight deformation of the cytometer channels and good hermeticity of the interface. We observed the profile of the cytometer channel and examined the amount of deformation under an optical microscope at different pressure. We took four photographs of the cytometer channel using a CCD camera as shown in Fig. 6, and we found the pressure range of 5-14 $\mathrm{kPa}$ would be appropriate for the packaging of the MGV chip. The other fluidic interconnects were also integrated on the upper block of the clamp, and $\mathrm{N}_{2}$ gas $(1-15 \mathrm{psi})$ were applied to press the deionized water into the MGV chip to test the hermeticity of the interface of PDMS/SU-8 and PDMS/Plexiglass.

A pneumatic system for the application of the pressure to the PDMS membrane of the MGV chip was set up for testing for the systematic study. DI water was loaded into the cytometer channel under a constant pressure of 4 psi.

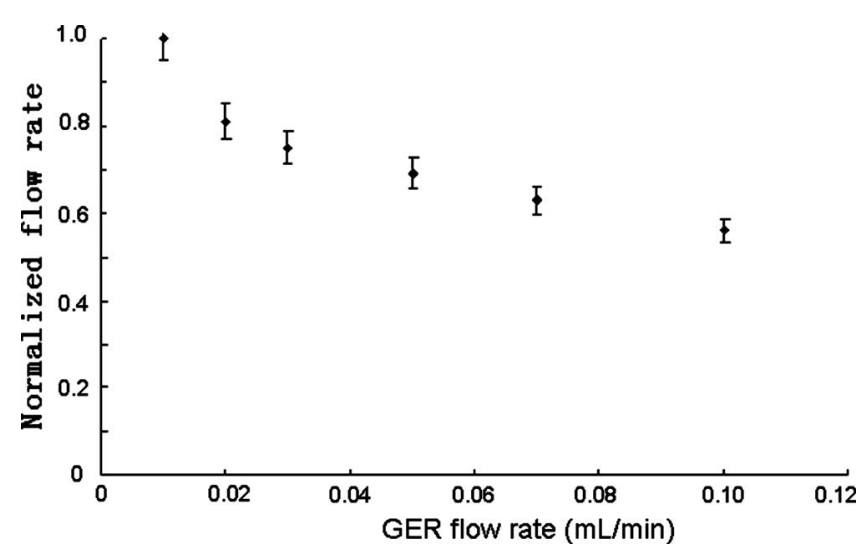

Fig. 8 Flow rate inside the cytometer channel as a function of the GER fluid flow rate inside the GER control channel.

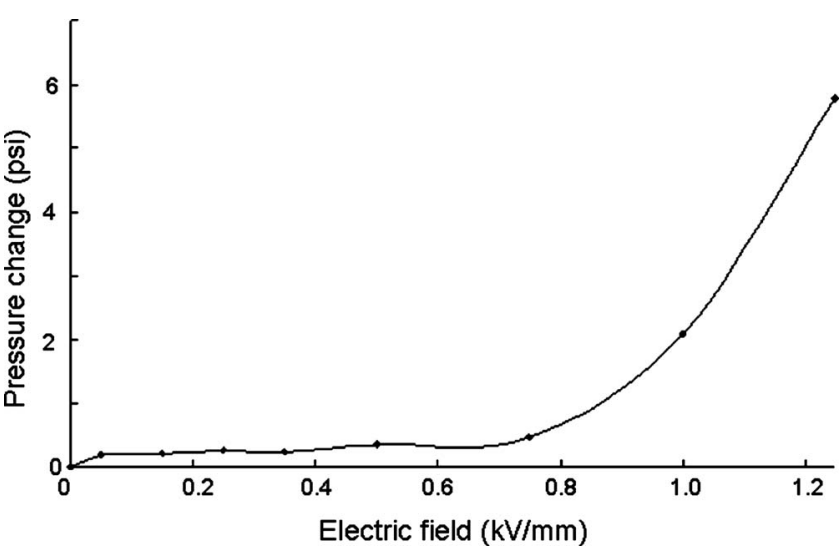

Fig. 9 Pressure increase as a function of input electric field inside the GER control channel.

The flow rate in the cytometer channel as a function of the input pressure in the GER control channel clearly showed the switching behavior of the valve (Fig. 7). To close the valve, a pressure of $>10$ psi was necessary.

Then, a similar experiment was done on the same MGV chip using the GER fluid control instead of the pneumatic system. Initially, the GER fluid was loaded into the GER control channel at the increasing flow rates using a syringe pump without the application of an electric field. The flow rate in the cytometer channel as a function of the GER fluid flow rate in the GER control channel is shown in Fig. 8. The flow rate of water in the cytometer channel was decreased when we increased the GER fluid flow rate. After the initial experiment, different electric field strengths were applied to the GER fluid to study the effect of ER fluid in the microchannel. Because of the optimization of the geometrical design of the SU-8 channels, the flow rate of the GER fluid was set to the constant value of $0.01 \mathrm{~mL} / \mathrm{min}$ in the following experiment. The flux of the GER fluid is only $10 \%$ of that used in our previous work. ${ }^{12}$

The pressure increase in the GER control channel and the switching behavior of water in the cytometer channel as a function of the electrical field applied are shown in Figs. 9 and 10, respectively. The pressure inside the GER control

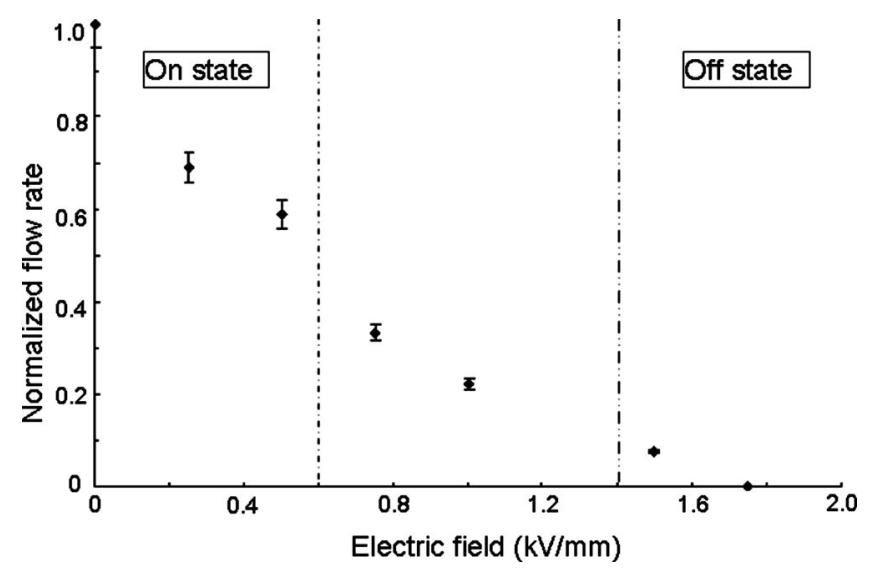

Fig. 10 Flow rate inside the cytometer channel as a function of input electric field inside the GER control channel. 


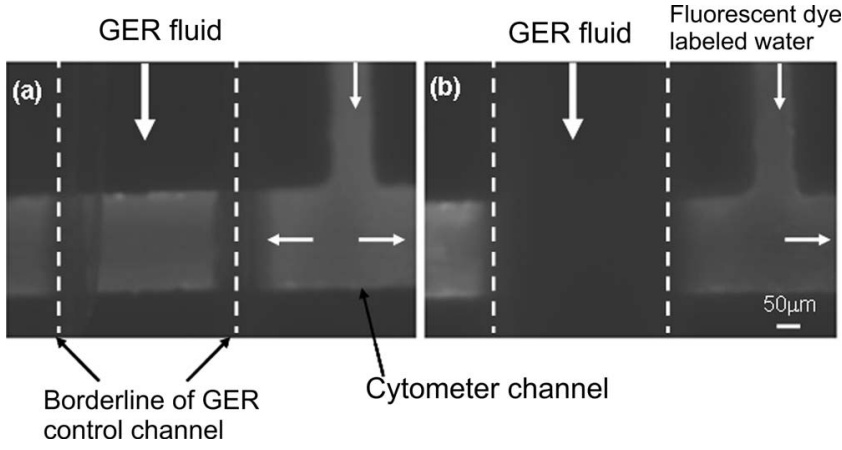

Fig. 11 Optical micrographs of the micro-GER valve: (a) on state and (b) off state.

channel gradually increased within the range of $0-800 \mathrm{~V} / \mathrm{mm}$, and it increased exponentially when the electrical field strength exceeded $800 \mathrm{~V} / \mathrm{mm}$. An electric field strength of $1250 \mathrm{~V} / \mathrm{mm}$ can result in an increase of more than 6 psi. When the electrical field strength was increased to $600 \mathrm{~V} / \mathrm{mm}$, the flow rate in the cytometer channel was measured to be only $\sim 50 \%$ of its previous value, and the flow rate was reduced to $<10 \%$ of its previous value when the electrical field strength was $1400 \mathrm{~V} / \mathrm{mm}$.

For electric field strengths much higher than $1250 \mathrm{~V} / \mathrm{mm}$, we observed clear morphology changes in the GER fluid in the microchannel with the help of fluorescence video microscopy (Olympus IX70 and Cohu CCD camera). The GER fluid can change from the liquid state to the solid state when the electric field exceeds $4000 \mathrm{~V} / \mathrm{mm}$, stopping the GER fluid flow in the control channel, as shown in Fig. 11. The on and off states in Fig. 11 are consistent with the results in Figs. 7 and 10. The flow of water labeled with fluorescent dye (Rhodamine 6G) in the cytometer channel was successfully stopped at an electrical field intensity of $2000 \mathrm{~V} / \mathrm{mm}$. For the recycling of the GER control layer, the PDMS layer can be peeled off, and the GER control channels can be cleaned several times with acetone and kept in a dry box for the next MGV operation.

\section{Conclusions}

A new micro-GER valve for microflow cytometry has been successfully designed and fabricated using four-mask MEMS technology. Three-dimensional microelectrodes and a GER control channel were integrated on a glass substrate by sputtering/electroplating and by SU- 8 photolithography instead of complicated and time-consuming fabrication processes.

Both permanent chemical bonding and mechanical sealing of the SU-8 and PDMS layers in the MGV were achieved and carefully studied. In addition, the electrorheological effects in this new MGV were characterized using fluorescence video microscopy. Both PDMS and SU-8 used in the MGV are biocompatible. Therefore, the valve is applicable for microfluidic control and can also be used for cell sorting in a microflow cytometer. This new micro-GER valve is promising for the precise manipulation of biofluids and bioparticles in various microfluidic systems and labs on chips.

\section{Acknowledgments}

This work was supported by a grant from the Hong Kong Research Grant Council (Grant No. 615970) and by a grant from the PROCORE-France/Hong Kong Joint Research Scheme sponsored by the RGC and the Consulate General of France in Hong Kong (Grant No. F-HK21/07T).

\section{References}

1. K. W. Oh, and C. H. Ahn, "A review of microvalves," J. Micromech. Microeng. 16(5), R13-R19 (2006).

2. D. R. Reyes, D. Iossifidis, P. A. Auroux, and A. Manz, "Micro total analysis systems. 1. Introduction, theory, and technology," Anal. Chem. 74(12), 2623-2918 (2002).

3. M. A. Unger, H. P. Chou, T. Thorsen, A. Scherer, and S. R. Quake, "Monolithic microfabricated valves and pumps by multilayer soft lithography," Science 288(7), 113-116 (2000).

4. Y. Xia and G. M. Whitesides, "Soft Lithography," Annu. Rev. Mater Sci. 28, 153-184 (1998).

5. G. B. Lee, C. H. Lin, and G. Chang, "Micro flow cytometers with buried SU-8/SOG optical waveguides," Sens. Actuators, A 103(30), 165-170 (2003).

6. E. Altendorf, E. Iverson, D. Schutte, B. Weigl, T. Osbom, R. Sabeti, and P. Yager, "Optical flow cytometry utilizing microfabricated silicon flow channels," Proc. SPIE 2678, 267-276 (1996).

7. S. Chung, S. J. Park, J. K. Kim, C. Chung, D. C. Han, and J. K. Chang, "Plastic microchip flow cytometer based on 2- and 3-dimensional hydrodynamic flow focusing," Microsyst. Technol. 9(8), 525-533 (2003).

8. S. Y. Yang, S. K. Hsiung, Y. C. Hung, C. M. Chang, T. L. Liao, and G. B. Lee, "A cell counting/sorting system incorporated with a microfabricated flow cytometer chip," Meas. Sci. Technol. 17(7), 20012009 (2006).

9. K. Yoshida, M. Kikuchi, J. H. Park, and S. Yokota, "Electrorheological behavior of suspension composed of titanium dioxide nanoparticles," Sens. Actuators, A 95, 227-233 (2002).

10. M. D. Volder, K. Yoshida, S. Yokota, and D. Reynaerts, "The use of liquid crystals as electrorheological fluids in microsystems model and measurements," J. Micromech. Microeng. 16, 612-619 (2006).

11. W. Wen, X. Huang, S. Yang, K. Lu, and P. Sheng, "The giant electrorheological effect in suspensions of nanoparticles," Nature Mater. 2(11), 727-730 (2003).

12. X. Niu, W. Wen, and Y. K. Lee, "Electrorheological-fluid-based microvalves," Appl. Phys. Lett. 87, 243501-243503 (2005).

13. X. Niu, L. Liu, W. Wen, and P. Sheng, "Active microfluidic mixer chip," Appl. Phys. Lett. 88, 153508-153511 (2006).

14. L. Liu, X. Chen, X. Niu, W. Wen, and P. Sheng, "Electrorheological fluid-based microfluidic pump," Appl. Phys. Lett. 89, 083505 (2006)

15. M. F. Spott, Design of Machine Element, Prentice Hall, Englewood Cliff., NJ (1998).

16. P. Rawlinson, Mater. World 7(5), 276-277 (1999).

Yun-Yang Ling received his BS and PhD degrees in the Department of Analytical Chemistry from Zhejiang University in 2000 and 2005 , respectively, and has been with Baptist University as a postdoctoral researcher from 2005 to 2007. In 2007, he moved to Hong Kong University of Science and Technology (HKUST) as a research associate in the Department of Mechanical Engineering. His current interests are microdevices and systems for chemical application and bioapplications.

Yechi Zhang received his BS in applied physics from Nanjing University in 2004. He worked as a process engineer in Oplink Communication Inc., Zhuhai, China. He obtained his MS in mechanical engineering from HKUST in Dec 2008. He worked as a research assistant for micro GER fluid project in Dr. Yi-Kuen Lee's laboratory at HKUST in 2007-2008. He is currently working as a materials engineer in the thermal group of Intel, Shenzhen, China. 
Ling et al.: Integrated microgiant electrorheological fluid valves...

Weijia Wen received his BS and MS in applied physics from Chongqing University, China in 1982 and 1988, respectively. He received his $\mathrm{PhD}$ in condensed matter and applied physics from the Institute of Physics, Chinese Academy of Science, Beijing, China in 1995. He worked as the postdoctoral researcher at HKUST from 1995 to 1997. Afterward, he moved to United States and worked as a postdoctoral researcher in the Department of Material Science and Engineering, UCLA from 1997 to 1999. Since 1999, he has been working in the Department of Physics, HKUST. His major research at HKUST focuses on soft condensed matter physics, electrorheological and magnetorheological fluids, field-induced pattern and structure transition, and thin-film physics.

Patrick Tabeling obtained his MS from the Ingénieur de l'Ecole Supérieure d'Electricite and DEA in Sciences des Matériaux Paris VI in 1974, his doctoral degrees (Thèse 3ème cycle) in 1976, and Thèse d'Etat in 1980. He worked as the research scientist in (LPS ENS Laboratoire de Physique Statistique de Ecole Normale Supéieure), Paris, from 1976 to 1990 . He worked as the postdoctoral researcher at Chicago University from 1984 to 1985 . In 1990, he became the directeur de recherches in LPS ENS. He also worked as a visiting professor in Prof. Chih-Ming Ho's lab, UCLA, from 1999 to 2000. In 2001, he became the director of the Microfluidics, MEMS \& Nanostructures Laboratory (MMN), Ecole Supérieure de Physique et de Chimie Industrielles, Paris. He is also a professor associated with École Polytechnique, France. In 1996, he received the Schlichting Award. He has coedited two books and authored one book about introduction to microfluidics, 80 refereed journal papers 30 proceeding papers, and 40 invited conferences.
Yi-Kuen Lee received his BS (with honor) in agricultural machinery engineering and $\mathrm{MS}$ in applied mechanics from the National Taiwan University, Taipei, Taiwan. He obtained his PhD in mechanical engineering with the MEMS major from the University of California, Los Angeles, in 2001. He has been an assistant professor in the Department of Mechanical Engineering, HKUST since 2001. He was promoted to associate professor in July 2007. He is associate director of Institute of Integrated Microsystem and Associate director of Center for Bioengineering and Biomedical Devices, HKUST. He is an active member of IEEE and ASME. His research interests include bio-MEMS, micro-nanofluidics, microelectroporation and electrofusion, micro-nanoelectrophoresis, micro/nano heat transfer. 